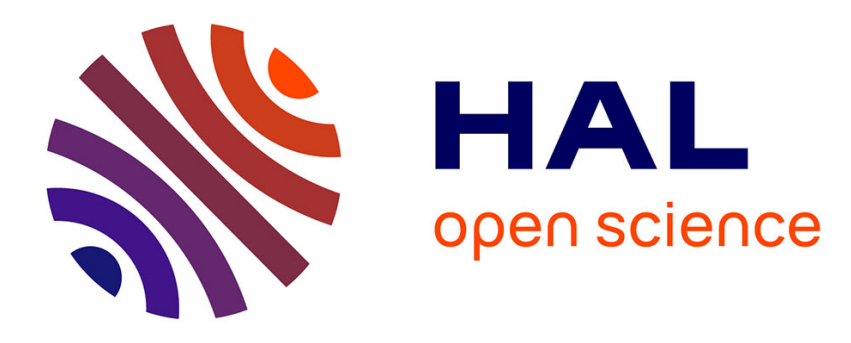

\title{
Nonlinear Modes of Vibration and Internal Resonances in Nonlocal Beams
}

Pedro Ribeiro, Olivier Thomas

\section{To cite this version:}

Pedro Ribeiro, Olivier Thomas. Nonlinear Modes of Vibration and Internal Resonances in Nonlocal Beams. Journal of Computational and Nonlinear Dynamics, 2017, 12 (3), pp.031017-1 - 031017-11. 10.1115/1.4035060 . hal-03166221

\section{HAL Id: hal-03166221 \\ https://hal.science/hal-03166221}

Submitted on 24 Jun 2021

HAL is a multi-disciplinary open access archive for the deposit and dissemination of scientific research documents, whether they are published or not. The documents may come from teaching and research institutions in France or abroad, or from public or private research centers.
L'archive ouverte pluridisciplinaire HAL, est destinée au dépôt et à la diffusion de documents scientifiques de niveau recherche, publiés ou non, émanant des établissements d'enseignement et de recherche français ou étrangers, des laboratoires publics ou privés. 


\author{
Pedro Ribeiro \\ DEMec/INEGI, \\ Faculdade de Engenharia, \\ Universidade do Porto, \\ R. Dr. Roberto Frias, $s / \mathrm{n}$ \\ Porto 4200-465, Portugal \\ e-mail: pmleal@fe.up.pt \\ Olivier Thomas \\ Arts et Métiers Paris Tech, \\ LSIS UMR CNRS 7296 \\ 8 Boulevard Louis XIV, \\ Lille 59046, France \\ e-mail: Olivier.THOMAS@ensam.eu
}

\section{Nonlinear Modes of Vibration and Internal Resonances in Nonlocal Beams}

\begin{abstract}
A nonlocal Bernoulli-Euler p-version finite-element $(p-F E)$ is developed to investigate nonlinear modes of vibration and to analyze internal resonances of beams with dimensions of a few nanometers. The time domain equations of motion are transformed to the frequency domain via the harmonic balance method (HBM), and then, the equations of motion are solved by an arc-length continuation method. After comparisons with published data on beams with rectangular cross section and on carbon nanotubes (CNTs), the study focuses on the nonlinear modes of vibration of CNTs. It is verified that the $p-F E$ proposed, which keeps the advantageous flexibility of the FEM, leads to accurate discretizations with a small number of degrees-of-freedom. The first three nonlinear modes of vibration are studied and it is found that higher order modes are more influenced by nonlocal effects than the first mode. Several harmonics are considered in the harmonic balance procedure, allowing us to discover modal interactions due to internal resonances. It is shown that the nonlocal effects alter the characteristics of the internal resonances. Furthermore, it is demonstrated that, due to the internal resonances, the nonlocal effects are still noticeable at lengths that are longer than what has been previously found.
\end{abstract} [DOI: 10.1115/1.4035060]

\section{Introduction}

Beams with dimensions of a few nanometers, or nanobeams, outstand due to their extremely small size and high-frequency dynamics $[1,2]$. There are diverse potential applications for nanobeams, including the detection and measurement of very small masses through shifts in oscillation frequency and in dynamic response, and nanoelectromechanic switches [2]. When vibrating at large amplitudes, nanobeams exhibit geometrical nonlinear behaviors that receive more and more attention, especially because one can take advantage of them to enhance the properties of the nanodevice [3-5].

The types of theoretical/numerical methods that have been more widely applied to investigate the vibrations of nanostructures can be divided in two large categories: atomistic/molecular methods [6,7] and continuum modeling approaches [7-21].

Atomistic modeling methods, such as molecular dynamics (MDs), have a high computational cost [6]. Formulations based on classical continuum elasticity theories do not have this disadvantage, but their application at the nanoscale is questionable $[1,15,22]$. Consequently, size-dependent continuum theories, which attempt to capture small-scale effects, have been proposed. One of these theories is the nonlocal elasticity theory of Eringen $[9,10]$, which has been applied to solve diverse problems at the nanoscale, including wave propagation in a periodic lattice structure and vibrations of nanobeams (e.g., see Refs. [7,12,14,19], and [21]). It takes into account scale effects and long-range atomic interactions. According to this theory, the stress at a reference point is not only a function of the strain at that point but also depends on strains at all the other points of the body. In the case of beams, the integral-partial differential equations of nonlocal elasticity can be reduced to partial differential equations [10]. The scale effect is considered in the differential constitutive equations as a material parameter, which can be obtained via experiments, molecular mechanics, or molecular dynamics.

A number of studies indicate that effects of smaller dimensions are captured by the nonlocal elasticity theory. Therefore, it can be applied to derive mathematical models for beams with reference dimensions of a few nanometers, avoiding the very large computational cost of discrete atomistic simulations. For example, according to Ref. [11], with a nonlocal beam Timoshenko model, it is possible to predict "the decrease of phase velocity when the wave number is so large that the microstructure of carbon nanotubes has a significant influence on the flexural wave dispersion." Another example is provided in Ref. [13], where a study is presented on flexural wave dispersion in double-walled carbon nanotubes, employing nonlocal elastic beam theories and MD simulations. The results reveal that the nonlocal beam model is able to predict the small-scale effect on flexural wave dispersion. In addition, Ansari and Sahmani [7] showed that nonlocal CNT models lead to values of natural frequencies that agree with the ones of MD simulations.

The natural modes of vibration provide information that is fundamental to understand the dynamic behavior of a beam. In conservative, geometrically nonlinear systems, free oscillations can be found, which are periodic and tend to the linear modes as the displacement amplitude decreases [23-25]. In this case, the harmonic motion with a constant shape becomes a periodic motion with variable shape. Not only the natural frequency of vibration changes with the vibration amplitude but also it is accompanied by its harmonics, hence the natural frequency is now the fundamental frequency of a periodic_-but not harmonic-oscillation. Nonlinear free vibrations of single-walled carbon nanotubes (SWCNTs) were studied in Ref. [14], employing Eringen's nonlocal elasticity theory. It was demonstrated that the small-scale affects the frequency and, less, the mode shapes of vibration in the nonlinear regime. Simsek [19] applied Eringen's nonlocal elasticity theory to investigate geometrically nonlinear free vibrations of beams. The motion was assumed to occur with a constant shape, equal to the one of the first linear mode shape of vibration. As in Ref. [14], it was found that the nonlocal parameter affects 
the frequencies of vibration in the nonlinear regime by increasing the degree of hardening spring. Both in Refs. [14] and [19], only the first mode of vibration was studied and it was assumed that the oscillations in the nonlinear regime are harmonic.

In this paper, the nonlinear modes of vibration of nonlocal beams are investigated, with most numerical tests focusing on carbon nanotubes. Since the study addresses modes of vibration of a fundamental element, i.e., single beams or CNTs, interactions with other structural elements and force fields are not considered. To carry out the investigations, a Bernoulli-Euler type $p$-version finite-element ( $p$-FE) is developed, where in a search for accurate discretizations in the spatial domain, the number of shape functions over the finite element(s) is increased. It is verified that this Bernoulli-Euler $p$-FE leads to accurate models-which accommodate variations of the mode shapes with the oscillation amplitude-with a small number of degrees-of-freedom. The equations of motion are passed to the frequency domain via the harmonic balance method (HBM), then they are solved by an arclength continuation method $[23,24]$. For the first time in this problem, several harmonics are considered in the HBM and, also originally, the evolution of the first three modes of vibration with the vibration amplitude is investigated. The consideration of several harmonics allows us to disclose previously undetected modal interactions-resulting in multimode, multifrequency vibrations-due to internal resonances in free vibrations. It is shown that the modes of vibration of nonlocal beams can be very different from the modes of local beams due to the internal resonances. Furthermore, it is also demonstrated that, because of internal resonances, the nonlocal effects are still noticeable at lengths that are longer than what has been previously found.

\section{Modeling and Analysis Approach}

2.1 Partial Differential Equations of Motion. Although the original constitutive relations of Eringen's nonlocal elasticity theory are integropartial differential equations [9], they were later simplified to second-order differential equations (Eq. (3.19) in Ref. [10]). In the Bernoulli-Euler beam case, only one differential constitutive equation is necessary; this equation is-as presented, for example, in Ref. [12]—as follows:

$$
\sigma_{x}(x, y, t)-\mu \frac{\partial^{2} \sigma_{x}(x, y, t)}{\partial x^{2}}=E \varepsilon_{x}(x, y, t)
$$

where $\sigma_{x}(x, y, t)$ is the axial stress, $\varepsilon_{x}(x, y, t)$ is the axial strain, $E$ is the Young modulus, and $\mu$ is a nonlocal parameter. The latter is $\mu=\left(e_{0} a\right)^{2}$, where $a$ represents an internal characteristic length (as lattice parameter or distance between $\mathrm{C}-\mathrm{C}$ bonds), and $e_{0}$ is a constant that depends on the material [10].

Following Bernoulli-Euler's beam theory, the longitudinal displacement, $\bar{u}(x, y, t)$, and the transverse displacement (in direction $y), \bar{v}(x, y, t)$, of any point of a beam are given by

$$
\left\{\begin{array}{l}
\bar{u}(x, y, t)=u(x, t)-y \frac{\partial v}{\partial x}(x, t) \\
\bar{v}(x, y, t)=v(x, t)
\end{array}\right.
$$

where $u(x, t)$ and $v(x, t)$ represent the displacements of points on the longitudinal axis $x$, an axis that contains the geometric centers of the cross sections; and $t$ represents the time.

To take into account the effect of large amplitude displacements, a Von Kármán type strain displacement relation is employed

$$
\varepsilon_{x}(x, y, t)=\frac{\partial u(x, t)}{\partial x}+\frac{1}{2}\left(\frac{\partial v(x, t)}{\partial x}\right)^{2}-y \frac{\partial^{2} v(x, t)}{\partial x^{2}}
$$

From Eqs. (1) and (3), we obtain the following relations for the longitudinal force and for the bending moment on a cross section:

$$
\begin{gathered}
N(x, t)-\mu \frac{\partial N^{2}(x, t)}{\partial x^{2}}=\mathrm{EA}\left(\frac{\partial u(x, t)}{\partial x}+\frac{1}{2}\left(\frac{\partial v(x, t)}{\partial x}\right)^{2}\right) \\
M(x, t)-\mu \frac{\partial^{2} M(x, t)}{\partial x^{2}}=\mathrm{EI} \frac{\partial^{2} v(x, t)}{\partial x^{2}}
\end{gathered}
$$

where $I$ represents the second moment of area of the cross sections about axes parallel to $z$.

Considering the free vibration case and neglecting the inertia due to cross section rotation, the partial differential equations of motion are

$$
\begin{gathered}
\frac{\partial N(x, t)}{\partial x}=\rho A \frac{\partial^{2} u(x, t)}{\partial t^{2}} \\
\frac{\partial^{2} M(x, t)}{\partial x^{2}}-\frac{\partial}{\partial x}\left(N(x, t) \frac{\partial v(x, t)}{\partial x}\right)=-\rho A \frac{\partial^{2} v(x, t)}{\partial t^{2}}+\rho I_{z} \frac{\partial^{4} v(x, t)}{\partial x^{2} \partial t^{2}}
\end{gathered}
$$

These are classical dynamic equilibrium equations, which also apply in the present nonlocal formulation [19]. The rotational inertia term of Eq. (7) will be neglected.

Neglecting the longitudinal inertia, Eq. (6) dictates that $N(x, t)$ is uniform along the beam (nevertheless, we will keep $x$ as an argument of the internal longitudinal force), so that Eq. (4) leads to

$$
N(x, t)=\mathrm{EA}\left(\frac{\partial u(x, t)}{\partial x}+\frac{1}{2}\left(\frac{\partial v(x, t)}{\partial x}\right)^{2}\right)
$$

and, returning to Eq. (6), one obtains

$$
\mathrm{EA} \frac{\partial}{\partial x}\left(\frac{\partial u(x, t)}{\partial x}+\frac{1}{2}\left(\frac{\partial v(x, t)}{\partial x}\right)^{2}\right)=0
$$

From Eqs. (5) and (7), one obtains the following expression for the bending moment:

$$
M(x, t)=\mathrm{EI} \frac{\partial^{2} v(x, t)}{\partial x^{2}}+\mu\left(-\rho A \frac{\partial^{2} v(x, t)}{\partial t^{2}}+\frac{\partial}{\partial x}\left(N \frac{\partial v(x, t)}{\partial x}\right)\right)
$$

With the bending moment as in Eq. (10), from Eq. (7) we arrive at

$$
\begin{aligned}
& \rho A\left(\frac{\partial^{2} v(x, t)}{\partial t^{2}}-\mu \frac{\partial^{4} v(x, t)}{\partial x^{2} \partial t^{2}}\right)+\mathrm{EI} \frac{\partial^{4} v(x, t)}{\partial x^{4}} \\
& +\mu \frac{\partial^{3}}{\partial x^{3}}\left(N(x, t) \frac{\partial v(x, t)}{\partial x}\right)-\frac{\partial}{\partial x}\left(N(x, t) \frac{\partial v(x, t)}{\partial x}\right)=0
\end{aligned}
$$

In terms of displacement components, the partial differential equations of motion are Eqs. (9) and (11), with $N(x, t)$ given by Eq. (8). These can be reduced, if one so wishes, to a nondimensional equation of motion, with the nondimensional nonlocal parameter $\zeta=e_{0} a / L$ as sole parameter [19]. $L$ represents the length of the beam.

2.2 Bernoulli-Euler $p$-Version Finite-Element Equations of Motion. In a Bernoulli-Euler type $p$-FE method, the displacement components-which are the unknowns of the present displacement based formulation—are written as

$$
\left\{\begin{array}{l}
u(\xi, t) \\
v(\xi, t)
\end{array}\right\}=\left[\begin{array}{cc}
\mathbf{f}_{u}(\xi)^{\mathrm{T}} & \mathbf{0} \\
\mathbf{0} & \mathbf{f}_{v}(\xi)^{\mathrm{T}}
\end{array}\right]\left\{\begin{array}{l}
\mathbf{q}_{u}(t) \\
\mathbf{q}_{v}(t)
\end{array}\right\}
$$

where $\mathbf{q}_{u}(t)$ and $\mathbf{q}_{v}(t)$ are the vectors of generalized coordinates in Lagrangian mechanics; since they specifically relate to 
displacement components, we will call them generalized displacements. Their second derivatives with respect to time, derivation represented by two dots, are the generalized accelerations. $\mathbf{f}_{u}(\xi)$ and $\mathbf{f}_{v}(\xi)$ are the vectors with shape functions. In the $p$-version FE, when one wishes to improve the approximation, one increases the number of shape functions and generalized coordinates, but does not refine the finite-element mesh. Because functions previously used are kept in improved approximations, the approach is said to be hierarchic [26]. This methodology has a number of advantages over traditional, $h$-version, finite elements, including the fact that it requires a small number of elements and a small number of degrees-of-freedom, while it keeps the versatility of the finite-element method.

The vector of shape functions $\mathbf{f}_{u}(\xi)$ is formed by linear functions plus a set of functions designated as $g$ functions in Ref. [24]; the set of shape functions $\mathbf{f}_{v}(\xi)$ is constituted by cubic polynomials plus the set of $f$ functions presented, for example, in Ref. [24]. The low order shape functions-linear functions of $\mathbf{f}_{u}(\xi)$ and cubic functions of $\mathbf{f}_{v}(\xi)$-are employed to respect geometric boundary conditions and to connect elements. At the element ends, the value of higher-order shape functions of the $g$ type is zero. In the same locations, both the higher-order shape functions of the $f$ type and their derivatives are zero. Therefore, higherorder shape functions do not interfere with geometric boundary conditions or with the connection between elements.

Nondimensional coordinate $\xi$, which varies from -1 to 1 , was introduced in Eq. (12). In all the case studies of this paper, the beam is represented by a single element and $x=\xi L / 2$. A single $p$-FE can be applied to analyze beams with diverse boundary conditions, including clamped-free [27,28], provided that the displacements and cross section rotations are not too large. In the latter case, more than one $p$-element and updating of the local reference frames would be necessary.

Inserting the expansion for $u(\xi, t)$ and $v(\xi, t)$ in the partial differential equation of motion (11), multiplying by each shape function of vector $\mathbf{f}_{v}(\xi)$, and integrating by parts in the domain, one obtains a set of ordinary differential equations of motion. This set can be written as follows:

$$
\begin{aligned}
& {\left[\mathbf{M}_{v}+\mathbf{M}_{\mu v}\right] \ddot{\mathbf{q}}_{v}(t)+\mathbf{K}_{v}^{0} \mathbf{q}_{v}(t)+\left[2 \mathbf{K}_{u v}^{1 \mathrm{~T}}\left(\mathbf{q}_{v}(t)\right)+\mathbf{K}_{\mu u v}^{1}\left(\mathbf{q}_{v}(t)\right)\right] \mathbf{q}_{u}(t)} \\
& \quad+\left[\mathbf{K}_{v}^{2}\left(\mathbf{q}_{v}(t)\right)+\mathbf{K}_{\mu v}^{2}\left(\mathbf{q}_{v}(t)\right)\right] \mathbf{q}_{v}(t)=\mathbf{0}
\end{aligned}
$$

$\mathbf{M}_{v}$ is the local mass matrix associated with the transverse acceleration, and $\mathbf{M}_{\mu v}$ is a mass matrix due to the effects of transverse inertia on the nonlocal part of the bending moment. The letters given as subscripts in the stiffness matrices $\mathbf{K}$ indicate the displacement components related to the diverse matrices or the nonlocal parameter. The numbers presented as superscripts indicate the dependence of the matrices on the transverse generalized displacements: 0 for a constant matrix, 1 for a matrix that is a linear function of $\mathbf{q}_{v}(t)$, and 2 for quadratic dependence. Therefore, matrix $\mathbf{K}_{v}^{0}$ is the constant stiffness matrix due to bending; matrix $\mathbf{K}_{u v}^{1}\left(\mathbf{q}_{v}(t)\right)$ is a matrix due to coupling between the longitudinal and the transverse displacement components and depends linearly on $\mathbf{q}_{v}(t)$; and matrix $\mathbf{K}_{v}^{2}\left(\mathbf{q}_{v}(t)\right)$ depends quadratically on $\mathbf{q}_{v}(t)$ and is due to the transverse displacement and to the consideration of large displacements. The former stiffness matrices are given in Ref. [24].

Because of the nonlocal and geometrically nonlinear effects, two new stiffness matrices appear in Eq. (13): $\mathbf{K}_{\mu u v}^{1}\left(\mathbf{q}_{v}(t)\right)$ and $\mathbf{K}_{\mu \nu}^{2}\left(\mathbf{q}_{v}(t)\right)$. These two matrices and matrix $\mathbf{M}_{\mu v}$ are given in the Appendix.

Substituting the displacement components given by expansion (12) in Eq. (9) and applying the weighted residuals method over one element, with shape functions of vector $\mathbf{f}_{u}(\xi)$ as weight functions, one obtains the following algebraic equation:

$$
\mathbf{K}_{u}^{0} \mathbf{q}_{u}(t)+\mathbf{K}_{u u}^{1}\left(\mathbf{q}_{v}(t)\right) \mathbf{q}_{v}(t)=\mathbf{0}
$$

Matrix $\mathbf{K}_{u}^{0}$ is a constant stiffness matrix due to longitudinal deformation without accounting for nonlinear effects, and matrix $\mathbf{K}_{u v}^{1}\left(\mathbf{q}_{v}(t)\right)$ is a matrix due to coupling between the longitudinal and the transverse displacement components, which depends linearly on $\mathbf{q}_{v}(t)$. These two matrices are given in Ref. [24].

The generalized displacements $\mathbf{q}_{u}(t)$ can be written as functions of $\mathbf{q}_{v}(t)$, using Eq. (14). In this way, one obtains the following ordinary differential equation of motion solely in $\mathbf{q}_{v}(t)$ from Eq. (13):

$$
\begin{aligned}
& {\left[\mathbf{M}_{v}+\mathbf{M}_{\mu v}\right] \ddot{\mathbf{q}}_{v}(t)+\mathbf{K}_{v}^{0} \mathbf{q}_{v}(t)-\mathbf{K}_{\mu u v}^{1}\left(\mathbf{q}_{v}(t)\right) \mathbf{K}_{u}^{0-1} \mathbf{K}_{u v}^{1}\left(\mathbf{q}_{v}(t)\right) \mathbf{q}_{v}(t)} \\
& \quad+\left[\mathbf{K}_{v}^{2}\left(\mathbf{q}_{v}(t)\right)+\mathbf{K}_{\mu v}^{2}\left(\mathbf{q}_{v}(t)\right)-2 \mathbf{K}_{u v}^{1 \mathrm{~T}}\left(\mathbf{q}_{v}(t)\right) \mathbf{K}_{u}^{0-1}\right. \\
& \left.\quad \mathbf{K}_{u v}^{1}\left(\mathbf{q}_{v}(t)\right)\right] \mathbf{q}_{v}(t)=\mathbf{0}
\end{aligned}
$$

When applying the harmonic balance method, this reduction allows us to automatically take into consideration the fact that for each vibration cycle in $\mathbf{q}_{v}(t)$, there are two cycles in $\mathbf{q}_{u}(t)$.

The nonlinear modes of vibration to be obtained here are periodic solutions of equations of motion (15). Hence, the vector of generalized displacements can be replaced by its Fourier series

$$
\mathbf{q}_{v}(t)=\sum_{i=1}^{k}\left(\mathbf{u}_{2 i-1} \cos ((2 i-1) \omega t)\right)
$$

which is truncated to $k$ terms. Vectors $\mathbf{u}_{2 i-1}$ are the coefficients of the harmonics. Since only cubic type nonlinear terms exist in Eq. (15) and because we are not going to investigate symmetry breaking bifurcations in time, which would lead to even harmonics $[29,30]$, series (16) only includes odd harmonics. Furthermore, because there are no traveling waves in the case studies considered, and neither is energy dissipation, sin terms are not required in the Fourier expansion.

Applying the harmonic balance method, a system of nonlinear algebraic equations is obtained, with the fundamental frequency of vibration, $\omega$, and the coefficients of each harmonic, $\mathbf{u}_{2 i-1}$, as unknowns. If $k=5$ in Eq. (16), this system is

$$
\begin{aligned}
& \left(-\omega^{2}\left[\begin{array}{ccc}
\mathbf{M} & \mathbf{0} & \mathbf{0} \\
\mathbf{0} & 9 \mathbf{M} & \mathbf{0} \\
\mathbf{0} & \mathbf{0} & 25 \mathbf{M}
\end{array}\right]+\left[\begin{array}{ccc}
\mathbf{K}_{\ell} & \mathbf{0} & \mathbf{0} \\
\mathbf{0} & \mathbf{K}_{\ell} & \mathbf{0} \\
\mathbf{0} & \mathbf{0} & \mathbf{K}_{\ell}
\end{array}\right]\left\{\begin{array}{l}
\mathbf{u}_{1} \\
\mathbf{u}_{2} \\
\mathbf{u}_{3}
\end{array}\right\}\right. \\
& +\left\{\begin{array}{l}
\mathbf{F}_{1}\left(\omega, \mathbf{u}_{\mathrm{HB}}\right) \\
\mathbf{F}_{3}\left(\omega, \mathbf{u}_{\mathrm{HB}}\right) \\
\mathbf{F}_{5}\left(\omega, \mathbf{u}_{\mathrm{HB}}\right)
\end{array}\right\}=\left\{\begin{array}{l}
\mathbf{0} \\
\mathbf{0} \\
\mathbf{0}
\end{array}\right\}
\end{aligned}
$$

where each vector $\mathbf{F}_{i}\left(\omega, \mathbf{u}_{\mathrm{HB}}\right), i=1,3,5$, is given by

$$
\mathbf{F}_{i}\left(\omega, \mathbf{u}_{\mathrm{HB}}\right)=\frac{2}{T} \int_{0}^{T} \mathbf{K}_{n \ell}\left(\mathbf{q}_{v}(\mathrm{t})\right) \mathbf{q}_{v}(\mathrm{t}) \cos (\mathrm{i} \omega \mathrm{t}) \mathrm{dt}, i=1,3,5
$$

Matrix $\mathbf{K}_{n \ell}\left(\mathbf{q}_{v}(\mathrm{t})\right)$ in Eq. (18) contains all the matrices that give rise to nonlinear terms in Eq. (15).

Algebraic nonlinear system of equations (17) is solved by an arc-length continuation method [23].

\section{Numerical Tests and Analysis}

3.1 Summary of Convergence and Verification Analysis. In order to verify the nonlocal Bernoulli-Euler $p$-FE and the computational procedure implemented, several comparisons with data published by other authors were carried out. Our values for the natural frequencies, in the linear regime, of clamped-clamped and hinged-hinged CNTs and nonlocal beams agreed with the values 
published in Refs. [12,16-18], and [30-32]. Due to space limitations, just one example is shown here, the case of a hinged-hinged beam presented in Ref. [12] and considered by several authors. The following properties are given, without units, in Ref. [12]: $L=10, E=30 \times 10^{-6}, \nu=0.3$, and $\rho=1$. The cross section of this beam is rectangular, we assume that the thickness $(h)$ is equal to the width $(b)$. Table 1 presents a comparison between the nondimensional fundamental frequency of the approach here proposed, computed with discretizations indicated by the value of $p_{o}$, and results given in Refs. [12,16], and [17]. Data computed using two theories in Ref. [12] are shown: EBT, i.e., Bernoulli-Euler's theory, and TBT, Timoshenko's beam theory (results computed using two third-order-shear deformation approaches in Ref. [12] are similar to the ones computed by TBT). The values from Ref. [16] were computed with a Timoshenko beam approach. In Ref. [17], Rayleigh's beam theory (RBT) is adopted. The nondimensional natural frequency is given by

$$
\bar{\omega}_{i}=\omega_{i} L^{2} \sqrt{\frac{\rho A}{E I}}
$$

where suffix $i$ indicates the mode number. Ratio $L / h$ is varied, keeping $L$ constant. The present results are either very close or equal to the ones published. Furthermore, similar values were published in Refs. [18] and [31]. Five shape functions guarantee five digits accuracy, whatever the value of the nonlocal parameter $\mu$ is. We note that the fundamental frequency decreases as the nonlocal parameter increases, a behavior that is been already found by a few authors.

Additional convergence tests were performed and it was verified that the nonlocal Bernoulli-Euler $p$-FE requires a small number of degrees-of-freedom for accuracy. A successful verification of the geometrically nonlinear case, but local, part of the model and of the code that solves it, was achieved by carrying out the example of Table 3 in Ref. [24], where the natural frequencies of a clamped-clamped beam are given; similar results were also computed in Ref. [30]. Another comparison with published results in the geometrically nonlinear regime $[23,33,34]$, but on a hinged-hinged beam, was performed. The natural frequencies of the present work agreed with the ones in Refs. [23,33,34].

In order to investigate the degree of convergence of the nonlocal approach proposed, when it is applied in the geometrically nonlinear and nonlocal case, backbone curves (amplitudes of harmonics of transverse displacement versus frequency of vibration) of the first three modes of vibration of CNTs were computed with different numbers of shape functions. Due to space limitation, only the main conclusions of this convergence analysis are given here, figures are not shown. It was verified that a model with $p_{o}=11, p_{l}=15$ is extremely precise in the first mode of vibration,

Table 1 Nondimensional fundamental frequency of vibration $\bar{\omega}_{1}, L=10$, hinged-hinged beam

\begin{tabular}{cccccccl}
\hline \hline$L / h$ & $\mu$ & $p_{o}=5$ & $p_{o}=21$ & EBT [12] & TBT [12] & TBT [16] & RBT [17] \\
\hline 100 & 0 & 9.8696 & 9.8696 & 9.8696 & 9.8683 & 9.8679 & 9.87 \\
& 1 & 9.4159 & 9.4159 & 9.4159 & 9.4147 & 9.4143 & 9.4162 \\
& 2 & 9.0195 & 9.0195 & 9.0195 & 9.0183 & 9.0180 & 9.0197 \\
& 3 & 8.6693 & 8.6693 & - & - & 8.6678 & 8.6695 \\
& 4 & 8.3569 & 8.3569 & 8.3569 & 8.3558 & 8.3555 & 8.3571 \\
& 5 & 8.0761 & 8.0761 & 8.0761 & 8.0750 & - & 8.0762 \\
20 & 0 & 9.8696 & 9.8696 & 9.8696 & 9.8381 & 9.8281 & 9.8798 \\
& 1 & 9.4159 & 9.4159 & 9.4159 & 9.3858 & 9.3763 & 9.4238 \\
& 2 & 9.0195 & 9.0195 & 9.0195 & 8.9907 & 8.9816 & 9.0257 \\
& 3 & 8.6693 & 8.6693 & - & - & 8.6328 & 8.6741 \\
& 4 & 8.3569 & 8.3569 & 8.3569 & 8.3302 & 8.3218 & 8.3606 \\
& 5 & 8.0761 & 8.0761 & 8.0761 & 8.0503 & - & 8.0789 \\
\hline \hline
\end{tabular}

computing accurately all the harmonics. The same model also leads to accurate computation of the second nonlinear natural frequency of vibration, some differences were found in the fifth harmonic, but these were irrelevant in the solutions computed. In the case of the third mode of vibration, model $p_{o}=11, p_{l}=15$ initially gives values equal to the ones of other models, but at larger vibration frequencies and vibration amplitudes, more shape functions are required for convergence. Model $p_{o}=13, p_{l}=17$ and, better, model $p_{o}=15, p_{l}=19$ accompany model $p_{o}=17$, $p_{l}=20$ - used as reference-more closely. This example confirms a well-known, but on the other hand, sometimes neglected, fact: convergence in the computation of linear modes of vibration of nonlocal beams does not guarantee convergence in the nonlinear regime. Three characteristics of nonlinear beams explain this need for more shape functions as the vibration amplitude increases: (1) the shapes of the modes of vibration change with the vibration amplitude; (2) interactions with higher order modes can occur; and (3) longitudinal deformation and bending are coupled.

In what concerns convergence with the number of harmonics, the one harmonic approximation originates rather accurate results until a certain vibration magnitude in the first three modes of vibration, then it deviates from the other approaches. The differences are mostly due to internal resonances that the one harmonic approach fails to detect. In the case studies performed, solutions computed with the first two and the first three odd harmonics are close to each other. Nevertheless, the order of the internal resonance that can be studied is necessarily limited by the number of harmonics employed.

3.2 Properties of CNTs. Before proceeding to the main goal of this paper, which is to investigate how nonlocal effects influence the modes of vibration of beams in the nonlinear regime and their internal resonances, we would like to justify the properties chosen for the case studies. We intend to employ properties which somehow represent a real example at the nanoscale. Carbon nanotubes (CNTs) can not only be very small but are also appointed as having great potential for diverse applications $[6,15]$. Hence, we will take as research case CNTs and adopt properties based on the ensuing literature analysis.

In Ref. [35], the Young moduli of diverse CNT are measured by analyzing thermal vibrations; values from $0.40 \mathrm{TPa}$ to 4.15 $\mathrm{TPa}$ are obtained, being the average $1.8 \mathrm{TPa}$. The inner diameter increases from $1.0 \mathrm{~nm}$ to $6.6 \mathrm{~nm}$ and the outside diameter from $5.6 \mathrm{~nm}$ to $24.8 \mathrm{~nm}$; specimens with lengths up to $5.81 \mu \mathrm{m}$ were obtained. Wang et al. [36] measured the mechanical properties of CNTs of different origins, using transmission electron microscopy. For carbon nanotubes produced by pyrolysis, the modulus of Young varied between $23 \pm 2.7 \mathrm{GPa}$ and $32.1 \pm 3.5 \mathrm{GPa}$. If the CNTs were produced by an arc-discharge technique, Young's moduli from $0.2 \mathrm{TPa}$ to $1.2 \mathrm{TPa}$ were found. With the arcdischarge technique, the carbon nanotubes have diameters between 5 and $50 \mathrm{~nm}$, and lengths ranging from 1 to $20 \mu \mathrm{m}$; with the pyrolysis technique, the inner diameter attained a maximum of $27.8 \pm 1 \mathrm{~nm}$, the outer diameter a maximum of $45.8 \pm 1 \mathrm{~nm}$, and the maximum length was $5.7 \pm 0.5 \mu \mathrm{m}$.

Yang et al. [14] estimated Young's modulus of CNT from $1.1468 \mathrm{TPa}$ to $1.1621 \mathrm{TPa}$, using molecular mechanics simulations. The diameter varied from $0.391 \mathrm{~nm}$ to $0.861 \mathrm{~nm}$. The same authors compute the natural frequencies of CNTs with radius $r=0.313 \mathrm{~nm}$, length $L=5 \mathrm{~nm}$, effective tube thickness $h_{t}=0.34 \mathrm{~nm}$, Young's modulus $E=1.1556 \mathrm{TPa}$, and $e_{0} a / L=0.15$ (hence, $e_{0} a=0.75 \mathrm{~nm}$ ). Wang et al. [37] gave $\rho=2300 \mathrm{~kg} \mathrm{~m}^{-3}$ for the mass density of CNTs.

Li and Chou [38] established a link between structural mechanics and molecular mechanics and arrived at Young's modulus for single-walled CNTs that vary with the diameter and chirality of the tubes, but are not far from $E=1.0 \mathrm{TPa}$. The authors assume "the thickness of a single-walled carbon nanotube to be the same as the interlayer spacing of graphite": $0.34 \mathrm{~nm}$. Xiao et al. [39] 
used the same effective thickness and also arrived at Young's modulus that are not far from $E=1.0 \mathrm{TPa}$. In both papers, reference is made to experimental analyses that somehow support the values obtained. Yoon et al. [40] gave a value of $1 \mathrm{TPa}$ for the modulus of Young, $0.35 \mathrm{~nm}$ for the effective thickness of singlewalled carbon nanotubes, and $1.3 \times 10^{3} \mathrm{~kg} / \mathrm{m}^{3}$ for the mass density.

Șimșek [19] adopted $e_{0} a$ between 0 and $2 \mathrm{~nm}$ and wrote that according to Wang [41], this is a "conservative estimate" of the nonlocal parameter $e_{0} a$ for SWCNTs. This estimate by Wang is based on references given in Ref. [41], according to which the measured frequency of SWNTs is greater than $10 \mathrm{THz}$. The estimates of Wang [41] are also referred/adopted in Ref. [15]. Ansari and Sahmani [7] proposed values for the nonlocal parameter for CNTs, by comparing the natural frequencies computed employing diverse beam theories with results of molecular dynamics simulations. The final values depended on the beam theory, the chirality of the CNT, and the boundary conditions. The lowest value presented in a table in Ref. [7] is $\mu=0.19\left(e_{0} a \cong 0.436 \mathrm{~nm}\right.$, Timoshenko beam, simply supported), and the largest value is $\mu=1.42$ ( $e_{0} a \cong 1.19 \mathrm{~nm}$, Bernoulli-Euler beam, clamped-clamped).

As seen in the former paragraphs, a large range of values can be found in the literature for material and geometric properties of CNTs. Based on the aforementioned publications, we can say that the values of Table 2 are appropriate for our study and these will be the properties adopted in the numerical tests that follow. To estimate the importance of nonlocal effects, $e_{0} a$ and $L$ will assume values in the ranges indicated in the table. More specifically, three lengths are considered- $L=25, L=50$, and $L=100 \mathrm{~nm}$ - and three values are attributed to $e_{0} a: 0,1$, and $2 \mathrm{~nm}$. The value of the nondimensional, nonlocal parameter $\zeta=e_{0} a / L$ is also given in the examples that follow and it increases from 0 to 0.08 .

Table 3 gives the natural frequencies of CNTs with properties written in Table 2, computed with $p_{o}=11$. The two boundaries are clamped, as they will be in the remaining test cases. The shorter CNT is, naturally, the one where the linear natural frequencies change the most with the nonlocal parameter $e_{0} a$. With $e_{0} a$ equal to $1 \mathrm{~nm}$, the first natural frequency decreases about $1 \%$, and the fifth natural frequency more than $16 \%$. If $e_{0} a$ is equal to 2 $\mathrm{nm}$, then the first natural frequency decreases about $3.7 \%$ and the fifth natural frequency a hefty $39 \%$. When $L=50 \mathrm{~nm}$ and $e_{0} a$ equal to $1 \mathrm{~nm}$, the first natural frequency decreases $0.25 \%$ and the fifth $4.9 \%$; with $e_{0} a$ equal to $2 \mathrm{~nm}$, the reductions are $1 \%$ and $16 \%$, respectively. The longer CNT is less affected by the nonlocal parameter, with modest variations in the lower natural frequencies. In comparison with the local value, the first two linear natural frequencies decrease only $0.26 \%$ and $0.91 \%$, respectively, when $e_{0} a$ becomes $2 \mathrm{~nm}$. But the linear natural frequencies of higher modes are more substantially altered, with the fifth linear natural frequency decreasing $4.9 \%$. Still for this length, when $e_{0} a=1 \mathrm{~nm}$, only the fifth natural frequency changes more than $1 \%$. As we will see in Sec. 3.3, larger sensitivity of higher order modes of vibration to nonlocal effects can be fundamental also at low frequencies of vibration, due to internal resonances induced by the geometrical nonlinearity.

3.3 Nonlinear Modes of Vibration and Internal Resonances in Nonlocal CNTs. The nonlinear modes of vibration of nonlocal CNTs are now investigated. All the data shown in this section were computed using the first three odd harmonics — since the first two even harmonics have zero amplitudes in the solutions
Table 3 Natural frequencies (Trad/s) in the linear regime, CNT of Table 2

\begin{tabular}{lllccccc}
\hline \hline$L(\mathrm{~nm})$ & $e_{0} a(\mathrm{~nm})$ & $\zeta$ & $\omega_{1}$ & $\omega_{2}$ & $\omega_{3}$ & $\omega_{4}$ & $\omega_{5}$ \\
\hline 25 & 0 & 0 & 0.3051 & 0.8411 & 1.649 & 2.726 & 4.072 \\
& 1 & 0.04 & 0.3022 & 0.8117 & 1.532 & 2.414 & 3.414 \\
& 2 & 0.08 & 0.2938 & 0.7388 & 1.290 & 1.882 & 2.485 \\
50 & 0 & 0 & 0.07628 & 0.2103 & 0.4122 & 0.6814 & 1.018 \\
& 1 & 0.02 & 0.07609 & 0.2084 & 0.4043 & 0.6592 & 0.9681 \\
& 2 & 0.04 & 0.07554 & 0.2029 & 0.3830 & 0.6036 & 0.8535 \\
100 & 0 & 0 & 0.01907 & 0.05257 & 0.1031 & 0.1704 & 0.2545 \\
& 1 & 0.01 & 0.01906 & 0.05245 & 0.1025 & 0.1689 & 0.2512 \\
& 2 & 0.02 & 0.01902 & 0.05209 & 0.1011 & 0.1648 & 0.2420 \\
\hline \hline
\end{tabular}

depicted-and $p_{o}=15, p_{l}=19$, or, exceptionally, more shape functions.

Figures 1-3 show backbone curves of CNTs with diverse lengths and diverse nonlocal parameters. In order to limit the number of figures, only the magnitude of the transverse vibration displacement at the beginning of a cycle is given as a function of the fundamental vibration frequency. This magnitude is represented by $V_{T}(\xi)$, with $\xi$ replaced by the nondimensional coordinate of the point where the magnitude is calculated and is divided by the diameter of the CNT, in order to provide an indication of the importance of geometrical nonlinear effects. $V_{T}(\xi)$ is computed by using Eqs. (12) and (16) with $t=0 \mathrm{~s}$, it is also the sum of the amplitudes - affected by the sign — of all the harmonics. At this stage, to turn the figures clearer, we start the continuation method at a linear mode of vibration and follow the path obtained by using a secant predictor, still without attempting to find additional branches. A more detailed analysis, with additional branches and bifurcations, is carried out after on two CNTs. To illustrate the effect of the nonlocal parameter on the dimension of the natural frequencies of vibration, it was decided to show the frequencies in radian per second. Other than the actual value of those frequencies and eventual convergence of the continuation code to other branches, curves with the same nondimensional nonlocal parameter $\zeta$ are identical.

Figures 1-3 show that the nonlocal parameters influence the backbone curves of the first three modes of vibration in diverse ways. First, as already seen, the nonlocal parameter affects the natural frequencies in the linear regime. Second, the larger the nonlocal parameter, the larger the degree of hardening (this had already been found, but solely in analyses of the first mode of vibration and assuming harmonic motions, in Refs. [14] and [19]). In our test cases, the changes in hardening with the nonlocal parameter are not very large. Third, changes in the nonlocal parameter lead to different inflexions or even turning points in the backbone curves; this phenomenon has not been found before. Addressing each figure separately, we start by noting that when $L=25 \mathrm{~nm}$, the effect of the nonlocal parameter is rather obvious in the three modes studied (Fig. 1). When $L=50 \mathrm{~nm}$, the effect of the nonlocal parameter is still visible in the three backbone curves (Fig. 2), although it is small in the first mode of vibration. In the longer CNT, $L=100 \mathrm{~nm}$ (Fig. 3), the backbone curve of the first mode barely changes with the nonlocal parameter. The backbone curves of the second mode computed with different nonlocal parameters are similar at low vibration amplitudes, although the degrees of hardening slightly differ. More noticeable than the

Table 2 Properties of the CNTs

\begin{tabular}{lcccc}
\hline \hline $\begin{array}{l}\text { Modulus of } \\
\text { elasticity, } E(\mathrm{TPa})\end{array}$ & $\begin{array}{c}\text { Mass density, } \\
\rho\left(\mathrm{kg} \mathrm{m}^{-3}\right)\end{array}$ & $\begin{array}{c}\text { Nonlocal parameter, } \\
e_{0} a(\mathrm{~nm})\end{array}$ & $\begin{array}{c}\text { Effective tube } \\
\text { thickness, } h_{t}(\mathrm{~nm})\end{array}$ & $\begin{array}{c}\text { Medium radius, } \\
r(\mathrm{~nm})\end{array}$ \\
\hline 1.0 & $1.3 \times 10^{3}$ & $0-2$ & 0.34 & $\begin{array}{c}\text { Length, } \\
L(\mathrm{~nm})\end{array}$ \\
\hline \hline
\end{tabular}




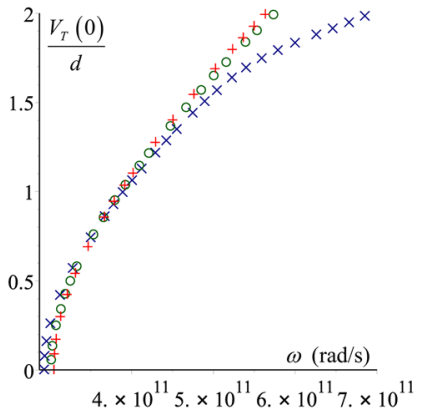

(a)

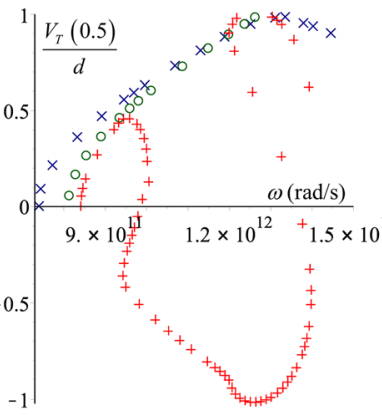

(b)

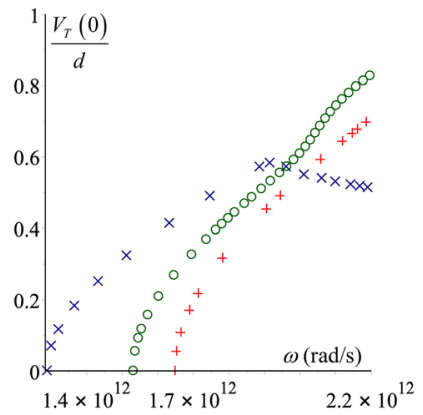

(c)

Fig. 1 Transverse vibration displacement in the beginning of a vibration cycle of the CNT with $L=25 \mathrm{~nm}$. The nonlocal parameter takes values: $+e_{0} a=0 \quad(\zeta=0), \bigcirc e_{0} a=1.0 \mathrm{~nm} \quad(\zeta=0.04)$, and $\times e_{0} a=2.0 \mathrm{~nm}(\zeta=0.08):(a)$ first mode, $(b)$ second mode, and $(c)$ third mode.

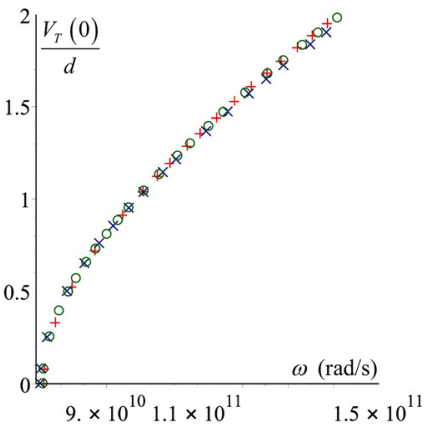

(a)

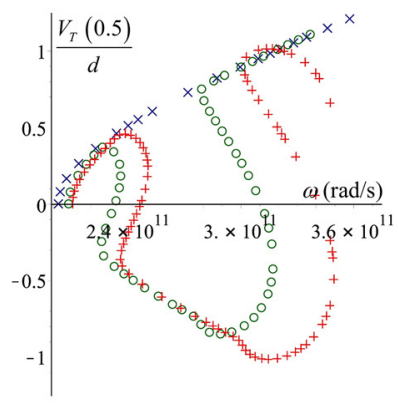

(b)

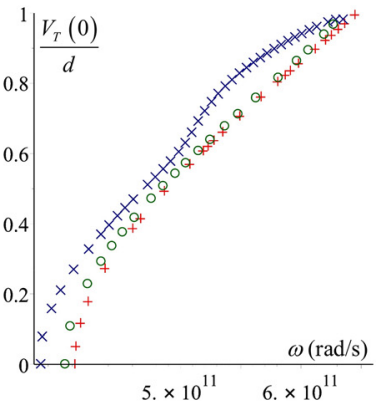

(c)

Fig. 2 Transverse vibration displacement in the beginning of a vibration cycle, of the CNT with $L=50 \mathrm{~nm}$. The nonlocal parameter takes values: $+e_{0} a=0 \quad(\zeta=0), \bigcirc e_{0} a=1.0 \mathrm{~nm} \quad(\zeta=0.02)$, and $\times e_{0} a=2.0 \mathrm{~nm}(\zeta=0.04):(a)$ first mode, $(b)$ second mode, and $(c)$ third mode.

different degrees of hardening is the fact that inflexion and turning points occur at vibration amplitudes and frequencies that depend upon the nonlocal parameter adopted. It results that the backbone curves of the second mode still depend very much on the nonlocal parameter when $L=100 \mathrm{~nm}$. In the case of the third mode, there is a small difference in the values of the natural frequencies and in the degree of hardening when $L=100 \mathrm{~nm}$.

Although we leave a deeper analysis of bifurcations to the following paragraphs, we should report that the locations of bifurcation points - detected by alterations in the sign of the determinant of a Jacobian matrix employed in the continuation method [23]are affected by the nonlocal parameter in all the modes of vibration and in all the CNTs analyzed in Figs. 1-3, including the longer CNT.

Now we analyze in more detail the CNT with length $L=50 \mathrm{~nm}$, considering the nonlocal effect with $e_{0} a=2 \mathrm{~nm}(\zeta=0.04)$ and not considering the nonlocal effect $\left(e_{0} a=0 \mathrm{~nm}\right)$. The backbone curves shown in Fig. 4 are related to the first nonlinear mode of vibration; the curves were computed with $p_{o}=15, p_{l}=19$ and three harmonics. The amplitude of harmonic number $i$ at point $\xi$ is represented by $V_{i}(\xi)$; this amplitude is affected by a plus or minus sign and divided by the external diameter of the CNT, $d$. The effect of the nonlocal parameter in the actual values, in radian per second, of the natural frequencies was shown in the previous paragraphs. In

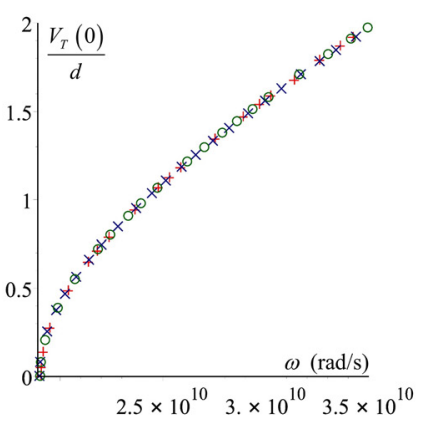

(a)

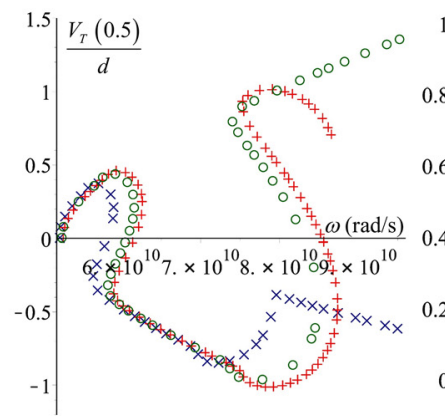

(b)

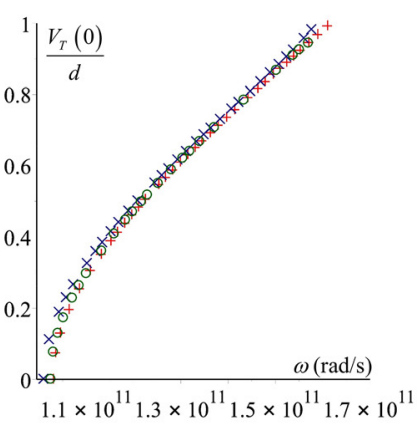

(c)

Fig. 3 Transverse vibration displacement in the beginning of a vibration cycle, of the CNT with $L=100 \mathrm{~nm}$. The nonlocal parameter takes values: $+e_{0} a=0 \quad(\zeta=0), \bigcirc e_{0} a=1.0 \mathrm{~nm}(\zeta=0.01)$, and $\times e_{0} a=2.0 \mathrm{~nm}(\zeta=0.02)$. 
Fig. 4 and in the figures that follow, in order to offer another perspective on the influence that the nonlocal parameter has on the backbone curves and to turn the comparison between degrees of hardening more direct, the horizontal axes are nondimensionalized by dividing the frequency of vibration by the linear natural frequency of vibration of the mode under analysis. Figure 4 shows that, when the length is equal to $50 \mathrm{~nm}$, the nonlocal parameter has a small influence on some solutions of the first mode, limited to slightly increasing hardening. On the other hand, the nonlocal parameter has a strong influence on modal interactions, greatly affecting turning points. With and without nonlocal effects, there is an excitation of the third mode of vibration associated with the fifth harmonic, due to a 1:5 resonance. However, when the nonlocal parameter is zero, this internal resonance affects the main branch slightly after $\omega / \omega_{\ell_{1}}=1.1$; if the nonlocal parameter $e_{0} a$ is $2 \mathrm{~nm}$, the 1:5 internal resonance occurs earlier, before $\omega /$ $\omega_{\ell_{1}}=1.024$. Furthermore, close to $\omega / \omega_{\ell_{1}}=1.8$, a bifurcation occurs when $e_{0} a=2 \mathrm{~nm}$; this bifurcation is not visible, in the range of frequencies portrayed, in the local model $\left(e_{0} a=0 \mathrm{~nm}\right)$. The latter bifurcation is due to the excitation of the fourth mode of vibration and is mainly linked with the fifth harmonic, although the first and third harmonics are also meaningful in the oscillations due to this bifurcation. The bifurcations are altered by the nonlocal parameter $e_{0} a$, because the relations between the natural frequencies of the first and higher modes vibration change with $e_{0} a$.

Figure 5 shows bifurcation diagrams obtained by starting the continuation at the second linear mode of vibration. Initially, the backbone curves of the two CNTs are not very different, with oscillations dominated by the first harmonic and the nonlocal CNT (in this case with $e_{0} a=2 \mathrm{~nm}, \zeta=0.04$ ) experiencing only slightly more hardening than the local CNT. But the bifurcations (here, term "bifurcation" includes bifurcations of the turning point type) are quite different in the local and nonlocal CNTs and they lead to rather distinct oscillations, which are reflected in Fig. 5 by the different relations between the vibration frequencies and the amplitudes of the harmonics.

Figures 6 and 7 allow us to further compare the behavior of the local and nonlocal CNTs, by analyzing the vibrations that correspond to point $P_{1}$ in Figs. 5(a) and 5(b). In this point, the first harmonic amplitude and the nondimensional frequency of vibration are the same in both (local and nonlocal) CNTs. The time histories and phase plane plots of Fig. 6 reflect the different harmonic contents of the displacements and velocities of the local and nonlocal CNTs. The strong presence of diverse harmonics, visible in this figure and in Fig. 5, indicates that we are in the presence of an internal resonance. The internal resonances are confirmed by the shapes assumed by the local and by the nonlocal CNTs

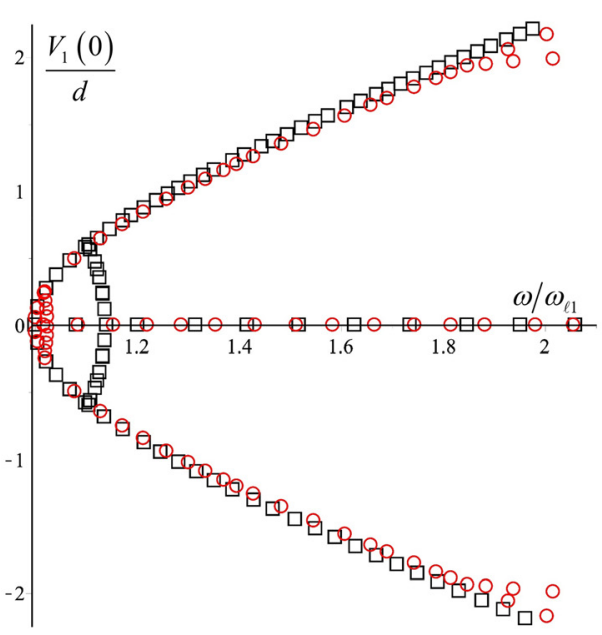

(a)

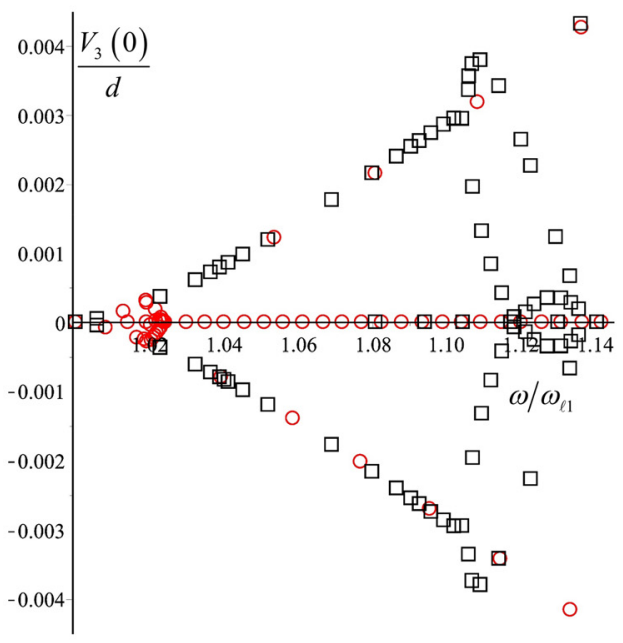

(c)

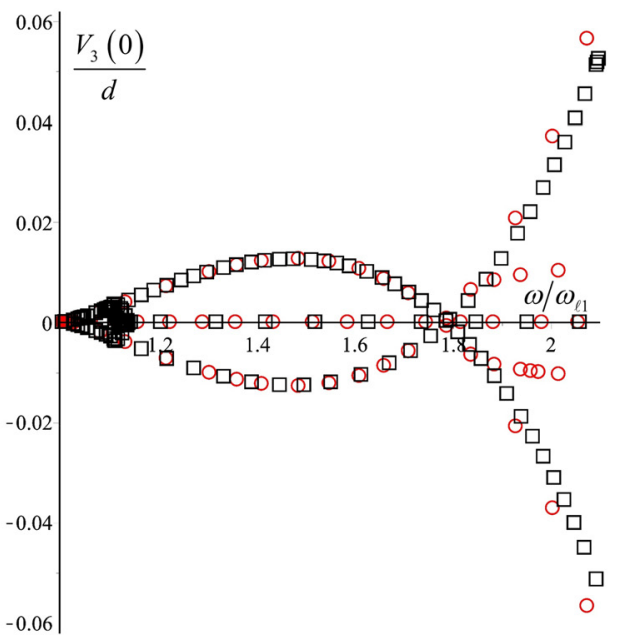

(b)

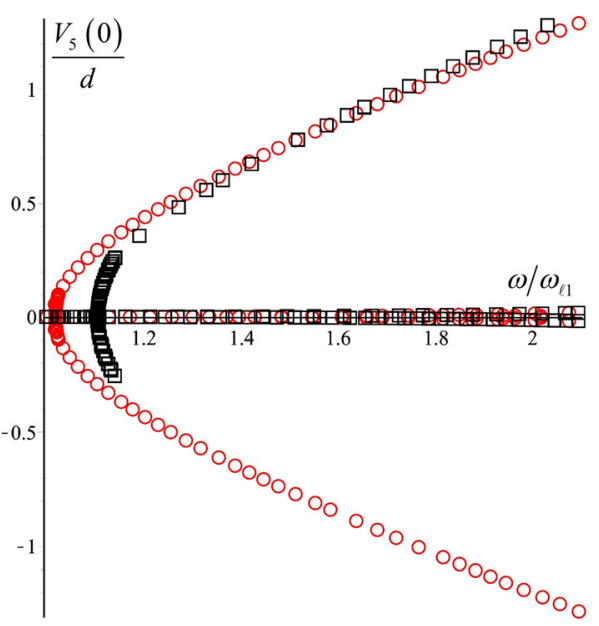

(d)

Fig. 4 First nonlinear mode and bifurcations; the figures show amplitudes of harmonics of the CNT with $L=50 \mathrm{~nm}$, when $e_{0} a=0 \mathrm{~nm} \square$ and when $e_{0} a=2.0 \mathrm{~nm}(\zeta=0.04) \bigcirc:$ (a) first harmonic, (b) third harmonic, $(c)$ detail of figure $(b)$, and (d) fifth harmonic 


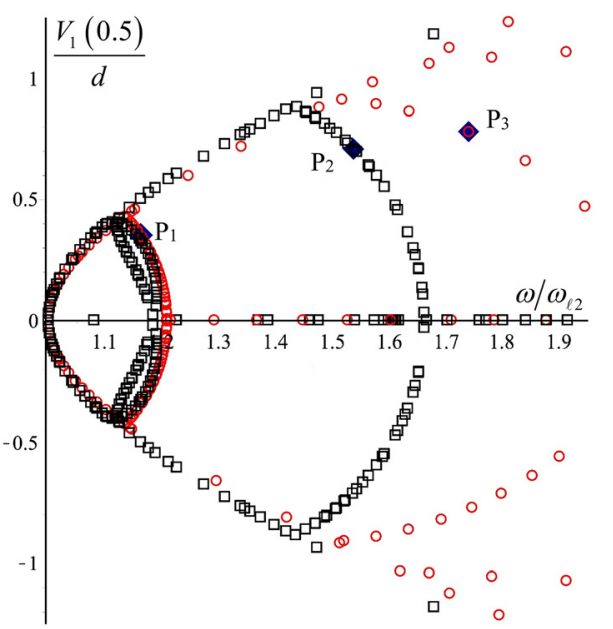

(a)

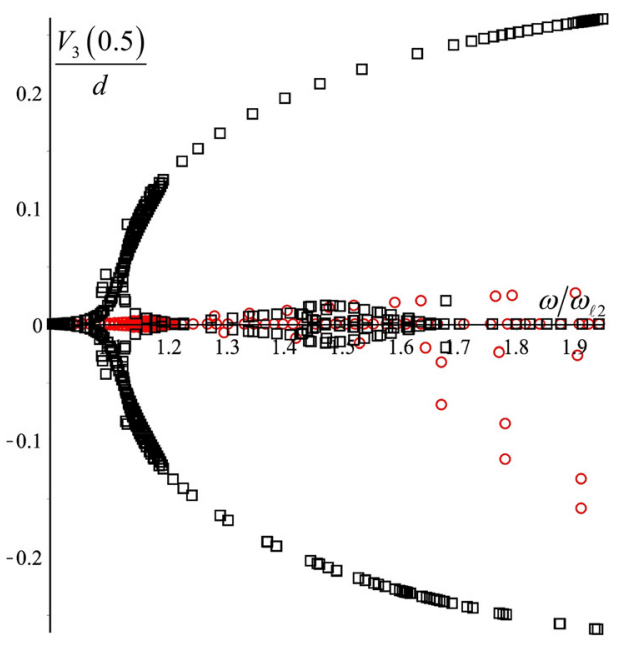

(c)

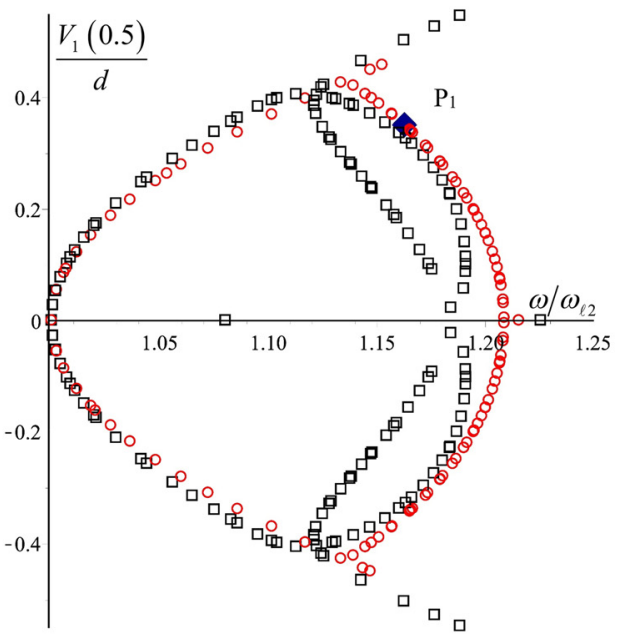

(b)

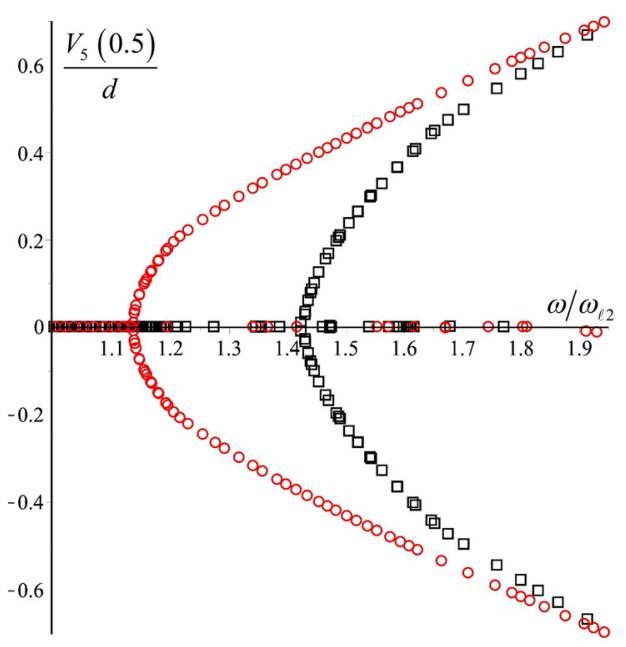

$(d)$

Fig. 5 Amplitudes of harmonics of the CNT with $L=50 \mathrm{~nm}$, second nonlinear mode and interactions, when $e_{0} a=0 \mathrm{~nm} \square$ and when $e_{0} a=2.0 \mathrm{~nm}(\zeta=0.04) \bigcirc$. The subfigures represent: $(a)-$ the first harmonic, $(b)$ - detail of figure (a), (c) - the third harmonic, and (d) - the fifth harmonic.

$\left(e_{0} a=2 \mathrm{~nm}\right.$ and $\left.\zeta=0.04\right)$, which are displayed in Fig. 7. Only half a cycle is shown and the shapes in the remaining part of the cycle are a repetition in inverse order of the ones displayed. It is obvious that more than one mode contributes to the oscillations of both CNTs. But the shapes assumed by the local CNT along the cycle are very different from the ones assumed by the nonlocal CNT. An analysis of the shapes assumed by each harmonic was also carried out (figures not shown). In point $P_{1}$ in Fig. 5, the first

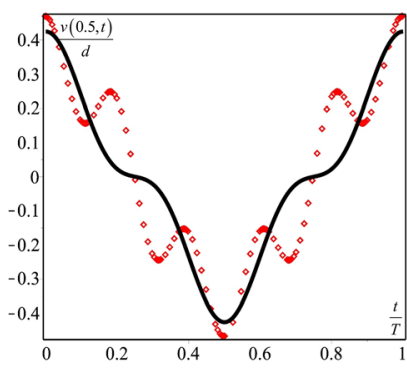

(a)

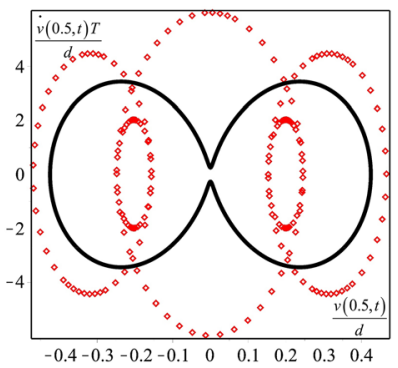

(b)
Fig. 6 Time histories (a) and phase plane plots $(b)$ of transverse oscillations of point $\xi=0.5$, local and nonlocal $\left(e_{0} a=2 \mathrm{~nm}\right.$ and $\zeta=0.04)$ CNTs, when $\omega / \omega_{12}=1.163$, and the nondimensional first harmonic amplitude is close to 0.33 (point $P_{1}$ in Fig. 5) harmonic is connected with the second mode of vibration in both CNTs; the third harmonic is related to the fourth mode of vibration in the local CNT, but in the nonlocal CNT the third harmonic barely appears and is influenced by more than one mode, including the sixth; the fifth harmonic is almost not excited in the local CNT, where the presence of the second and sixth modes is visible in connection with this harmonic; on the other hand, in the nonlocal CNT the fifth harmonic is essentially related to the sixth mode. So, in this example, not only the modes involved in the oscillations of the local and nonlocal CNTs are different but so are the harmonics that appear in the oscillations and the orders of the internal resonances (1:3 in the local CNT, 1:5 in the nonlocal).

Another comparative analysis of the behavior of the two CNTs is carried out by addressing points $P_{2}$ (local CNT) and $P_{3}$ (nonlocal CNT) in Fig. 5. Now, the solutions under analysis have in common the amplitude of the first harmonic, which is approximately $0.77 d$. The value of the nondimensional frequency of vibration $\omega / \omega_{\ell 2}$ is 1.51 in point $P_{2}$ and 1.74 in point $P_{3}$. This examination is performed with the help of Fig. 8, where shapes assumed by the local and nonlocal CNTs along half a vibration period are shown. The individual shapes of each harmonic were also inspected; again they are not shown here in order to limit the number of figures.

Both in solutions $P_{2}$ and $P_{3}$, more than one mode participates in the oscillations, with the first harmonic associated with the second 

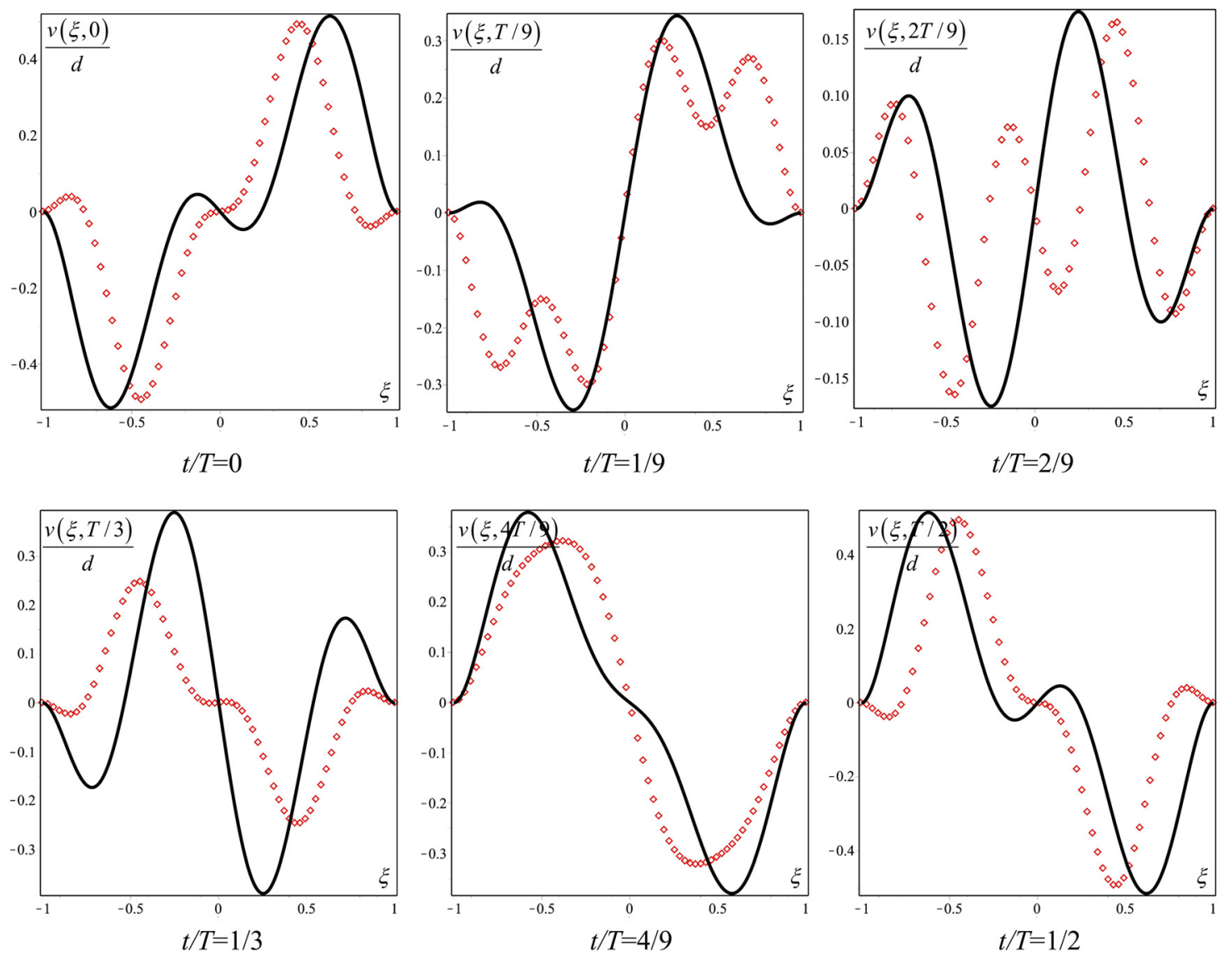

Fig. 7 Shapes assumed by local $(-)$ and nonlocal $\left(\diamond, e_{0} a=2 \mathrm{~nm}, \zeta=0.04\right)$ CNTs when $\omega / \omega_{12}=1.163$ and $V_{1} / d \cong 0.33$, point $P_{1}$ in Fig. 5, along half a vibration cycle

mode of vibration. The fifth harmonic is also important in both CNTs, although smaller than the first. In the nonlocal CNT, the fifth harmonic is connected with the seventh mode of vibration; in the local CNT, it is connected with the sixth mode. The third harmonic is about 1 order of magnitude smaller than the fifth in both CNTs and is affected by more than one mode of vibration; apparently, the second and seventh modes influence the third harmonic of the nonlocal CNT, and the second and sixth modes the third harmonic of the local CNT.

Finally, we note that a branch of solutions appears in Fig. 5 at larger vibration amplitudes only when nonlocal effects are taken into account; it has no counterpart in the local CNT, in the range of frequencies portrayed. This branch of solutions is due to an internal resonance. All the harmonics are present in these oscillations, with their importance decreasing as the order of the harmonics increases. It was verified that the first harmonic is still connected to the second mode of vibration, while the third and fifth harmonics are influenced by higher modes.

The analysis of this section shows that CNTs which are longer than what has been previously deducted-for example, according to Ref. [14], the nonlocal effects are negligible after $L>16 \mathrm{~nm}-$ may still be strongly influenced by the nonlocal effects: it is only required that a higher order mode of vibration is present in the oscillation, either directly or due to an internal resonance. Internal resonances can arise or cease to exist due to nonlocal effects; furthermore, when they occur both in the local and nonlocal cases, the modes and/or harmonics involved in internal resonances of local and nonlocal CNTs can be different. If we remember that nodes of vibration exist in higher order modes, it is immediately understood that the distance between the nodes of the highest mode involved in the vibration, not the CNT length, will dictate how important the nonlocal effects are.

\section{Conclusions}

A beam p-version type finite-element, based on Bernoulli-Euler's hypothesis and on Eringen's nonlocal theory of elasticity, was presented. The ordinary differential equations of motion in the time domain were transformed to the frequency domain, for the first time in this problem with the help of a multiharmonic harmonic balance method. The last equations were solved by a continuation method.

A few tests were carried out in the linear and nonlinear regimes. The Bernoulli-Euler $p$-finite version element ( $p$-FE) implemented here provided results that agreed with published data; furthermore, convergence analyses were performed and it was verified that the nonlocal $p$-FE does not require a large number of degreesof-freedom for accuracy. It was verified that the nonlocal parameter reduces the natural frequencies in the linear regime and, in the nonlinear regime, the larger the nonlocal parameter, the larger the degree of hardening; the latter effect was not very pronounced in the test cases of this paper. The reason why the nonlocal effects reduce the natural frequencies in the linear regime is that the inertia increases, as expressed in the mathematical model by an additional mass matrix. On the other hand, the part of the longitudinal force that is due to the large displacement increases with the "nonlocality" that is with the consideration of strains in the complete domain; hence, the hardening spring effect is larger in nonlocal than in local beams.

The first three nonlinear modes of vibration of CNTs modeled as nonlocal Bernoulli-Euler beams were analyzed. In addition to the investigation of higher order modes, the main original findings of this study are related to internal resonances. In the absence of internal resonances, higher order modes of vibration are-both in the linear and nonlinear regimes-more altered by nonlocal 

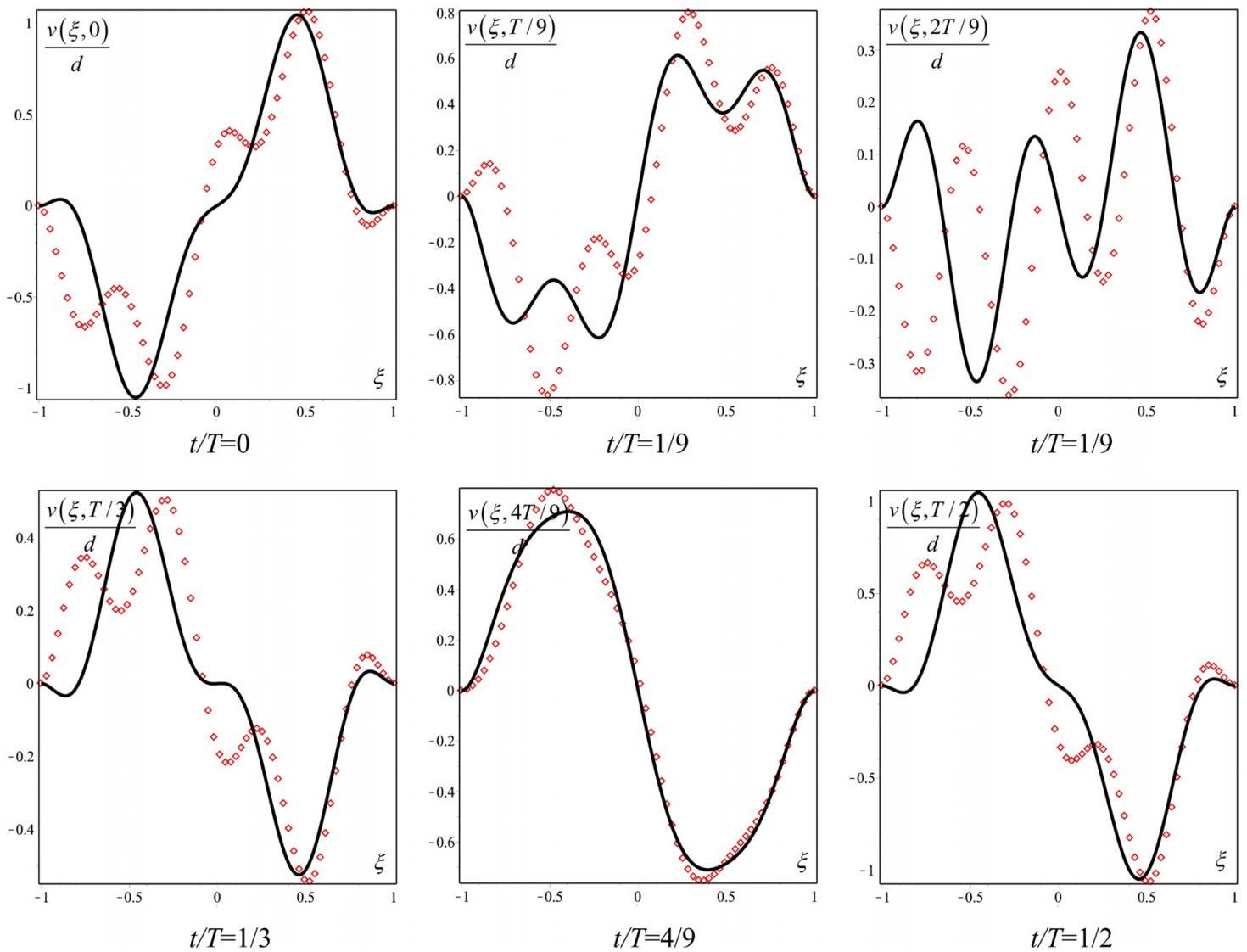

Fig. 8 Shapes assumed along half a vibration cycle by local $(-)$ and nonlocal $\left(\diamond, e_{0} a=2 \mathrm{~nm}, \zeta=0.04\right)$ CNTs, solutions corresponding, respectively, to points $P_{2}$ and $P_{3}$ of Fig. 5

effects than the first mode is; this occurs because the length that dictates the importance of nonlocal effects is not the total length of the beam or CNT, but the distance between nodes of vibration. It was found that different nonlocal parameters cause different inflections, bifurcation, and turning points in the backbone curves of any mode, including the first. So, also in the first mode of vibration, it was found that the vibrations of CNTs that are not extremely short (for example, with a length equal to $50 \mathrm{~nm}$ and nonlocal parameter $e_{0} a=2.0 \mathrm{~nm}$, or, without dimensions, $\zeta=0.04)$ can be radically changed by the nonlocal effects. The main explanation for this is that the nonlinearity promotes interaction with higher order modes, which, as said, are more modified than low order modes by nonlocal effects. Nonlocal effects alter the frequencies at which internal resonances occur and nonlocal effects can change the modes involved in the internal resonances. These findings complement analysis by other authors, who assumed harmonic motion and therefore could not reveal the importance of internal resonances.

\section{Acknowledgment}

The first author acknowledges the contribution provided by $\mathrm{Mr}$. Mário Oliveira in the beginning of this work and the financial support of the Portuguese Science and Technology foundation (FCT), under sabbatical Grant No. SFRH/BSAB/1442/2014.

\section{Appendix: Bernoulli-Euler $p$-FE Matrices Due to Nonlocal Effects}

The transverse inertia affects the second derivative of the bending moment with respect to axis $x$ (see Eq. (7)), which appears in the nonlocal constitutive equation. From here results the following nonlocal mass matrix of the Bernoulli-Euler $p$-FE:

$$
\mathbf{M}_{\mu v}=\rho A \mu \frac{2}{L} \int_{-1}^{1} \frac{\partial \mathbf{f}_{v}(\xi)}{\partial \xi} \frac{\partial \mathbf{f}_{v}(\xi)^{\mathrm{T}}}{\partial \xi} \mathrm{d} \xi
$$

One of the stiffness matrices of the Bernoulli-Euler $p$-FE due to nonlocal and geometrical nonlinear effects is a linear function of the generalized transverse displacements; this matrix is as follows:

$$
\mathbf{K}_{\mu u v}^{1}\left(\mathbf{q}_{v}(t)\right)=\frac{16 \mathrm{EA} \mu}{L^{4}} \int_{-1}^{1} \frac{\partial^{2} \mathbf{f}_{v}(\xi)}{\partial \xi^{2}} \frac{\partial \mathbf{f}_{u}(\xi)^{\mathrm{T}}}{\partial \xi} \frac{\partial^{2} \mathbf{f}_{v}(\xi)^{\mathrm{T}}}{\partial \xi^{2}} \mathbf{q}_{v}(t) \mathrm{d} \xi
$$

The other stiffness matrix that appears due to the nonlocal effects and to the large displacements, a matrix which depends quadratically on $\mathbf{q}_{v}(t)$, is

$$
\left.\mathbf{K}_{\mu u v}^{2}\left(\mathbf{q}_{v}(t)\right)=\frac{16 \mathrm{EA} \mu}{L^{5}} \int_{-1}^{1} \frac{\partial^{2} \mathbf{f}_{v}(\xi)}{\partial \xi^{2}} \frac{\partial^{2} \mathbf{f}_{v}(\xi)^{\mathrm{T}}}{\partial \xi^{2}} \frac{\partial \mathbf{f}_{v}(\xi)^{\mathrm{T}}}{\partial \xi} \mathbf{q}_{v}(t)\right)^{2} \mathrm{~d} \xi
$$

\section{References}

[1] Eom, K., Park, H. S., Yoon, D. S., and Kwon, T., 2011, "Nanomechanical Resonators and Their Applications in Biological/Chemical Detection: Nanomechanics Principles," Phys. Rep., 503(4-5), pp. 115-163.

[2] Li, C., Thostenson, E. T., and Chou, T. W., 2008, "Sensors and Actuators Based on Carbon Nanotubes and Their Composites: A Review," Compos. Sci. Technol., 68(6), pp. 1227-1249.

[3] Karabalin, R. B., Masmanidis, S. C., and Roukes, M. L., 2010, "Efficient Parametric Amplification in High and Very High Frequency Piezoelectric Nanoelectromechanical Systems," Appl. Phys. Lett., 97(18), p. 183101.

[4] Lazarus, A., Thomas, O., and Deü, J. F., 2012, "Finite Element Reduced Order Models for Nonlinear Vibrations of Piezoelectric Layered Beams With Applications to NEMS," Finite Elem. Anal. Des., 49(1), pp. 35-51. 
[5] Nguyen, V. N., Baguet, S., Lamarque, C. H., and Dufour, R., 2015, "Bifurcation-Based Micro-/Nanoelectromechanical Mass Detection," Nonlinear Dyn., 79(1), pp. 647-662.

[6] Gibson, R. F., Ayorinde, E. O., and Wen, Y.-F., 2007, "Vibrations of Carbon Nanotubes and Their Composites: A Review," Compos. Sci. Technol., 67(1), pp. $1-28$.

[7] Ansari, R., and Sahmani, S., 2012, "Small Scale Effect on Vibrational Response of Single-Walled Carbon Nanotubes With Different Boundary Conditions Based on Nonlocal Beam Models," Commun. Nonlinear Sci. Numer. Simul., 17(4), pp. 1965-1979.

[8] Yang, F., Chong, A. C. M., Lam, D. C. C., and Tong, P., 2002, "Couple Stress Based Strain Gradient Theory for Elasticity," Int. J. Solids Struct., 39(10), pp. 2731-2743.

[9] Eringen, A. C., 1972, "Linear Theory of Nonlocal Elasticity and Dispersion of Plane Waves," Int. J. Eng. Sci., 10(5), pp. 425-435.

[10] Eringen, A., 1983, "On Differential Equations of Nonlocal Elasticity and Solutions of Screw Dislocation and Surface Waves," J. Appl. Phys., 54(9), p. 4703.

[11] Wang, L. F., and Hu, H. Y., 2005, "Flexural Wave Propagation in SingleWalled Carbon Nanotubes," Phys. Rev. B, 71(19), p. 195412.

[12] Reddy, J. N., 2007, "Nonlocal Theories for Bending, Buckling and Vibration of Beams," Int. J. Eng. Sci., 45(2-8), pp. 288-307.

[13] Hu, Y.-G., Liew, K. M., and Wang, Q., 2009, "Nonlocal Elastic Beam Models for Flexural Wave Propagation in Double-Walled Carbon Nanotubes," J. Appl. Phys., 106(4), p. 044301.

[14] Yang, J., Ke, L. L., and Kitipornchai, S., 2010, "Nonlinear Free Vibration of Single-Walled Carbon Nanotubes Using Nonlocal Timoshenko Beam Theory," Physica E, 42(5), pp. 1727-1735.

[15] Arash, B., and Wang, Q., 2012, "A Review on the Application of Nonlocal Elastic Models in Modeling of Carbon Nanotubes and Graphenes," Comput. Mater. Sci., 51(1), pp. 303-313.

[16] Thai, H.-T., 2012, "A Nonlocal Beam Theory for Bending, Buckling, and Vibration of Nanobeams," Int. J. Eng. Sci., 52, pp. 56-64.

[17] Eltaher, M. A., Alshorbagy, A. E., and Mahmoud, F. F., 2013, "Vibration Analysis of Euler-Bernoulli Nanobeams by Using Finite Element Method," Appl. Math. Modell., 37(7), pp. 4787-4797.

[18] Oliveira, M. A. M., 2014, "Vibrações de Nanoplacas," M.Sc. thesis, Universidade do Porto, Porto, Portugal.

[19] Şimşek, M., 2014, "Large Amplitude Free Vibration of Nanobeams With Various Boundary Conditions Based on the Nonlocal Elasticity Theory," Composites, Part B, 56, pp. 621-628

[20] Ghayesh, M. H., and Farokhi, H., 2016, "Coupled Nonlinear Dynamics of Geometrically Imperfect Shear Deformable Extensible Microbeams," ASME J. Comput. Nonlinear Dyn., 11(4), p. 041001.

[21] Houmat, A., 2016, "Nonlinear Free Vibration of Non-Prismatic Single-Walled Carbon Nanotubes by a Non-Local Shear Deformable Beam p-Element," Acta Mech., 227(4), pp. 1051-1065.

[22] Ghoniem, N. M., Busso, E. P., Kioussis, N., and Huang, H., 2003, "Multiscale Modelling of Nanomechanics and Micromechanics: An Overview," Philos. Mag., 83(31-34), pp. 3475-3528.
[23] Lewandowski, R., 1994, "Nonlinear Free-Vibrations of Beams by the FiniteElement and Continuation Methods," J. Sound Vib., 170(5), pp. 577-593.

[24] Ribeiro, P., and Petyt, M., 1999, "Non-Linear Vibration of Beams With Internal Resonance by the Hierarchical Finite-Element Method," J. Sound Vib., 224(4), pp. 591-624.

[25] Touzé, C., Thomas, O., and Huberdeau, A., 2004, "Asymptotic Non-Linear Normal Modes for Large-Amplitude Vibrations of Continuous Structures," Comput. Struct., 82(31-32), pp. 2671-2682.

[26] Meirovitch, L., and Baruh, H., 1983, "On the Inclusion Principle for the Hierarchical Finite Element Method,” Int. J. Numer. Methods Eng. Struct., 19(2), pp. 281-291.

[27] Stoykov, S., and Ribeiro, P., 2010, "Nonlinear Forced Vibrations and Static Deformations of 3D Beams With Rectangular Cross Section: The Influence of Warping, Shear Deformation and Longitudinal Displacements," Int. J. Mech. Sci., 52(11), pp. 1505-1521.

[28] Stoykov, S., and Ribeiro, P., 2013, "Vibration Analysis of Rotating 3D Beams by the p-Version Finite Element Method," Finite Elem. Anal. Des., 65, pp. 76-88.

[29] Ribeiro, P., 2009, "Asymmetric Solutions in Large Amplitude Free Periodic Vibrations of Plates," J. Sound Vib., 322(1-2), pp. 8-14.

[30] Stoykov, S., and Ribeiro, P., 2011, "Nonlinear Free Vibrations of Beams in Space Due to Internal Resonance,” J. Sound Vib., 330(18-19), pp. 4574-4595.

[31] dos Santos, J. V. A., and Reddy, J. N., 2012, "Vibration of Timoshenko Beams Using Non-Classical Elasticity Theories," Shock Vib., 19(3), pp. 251-256.

[32] Fu, Y. M., Hong, J. W., and Wang, X. Q., 2006, "Analysis of Nonlinear Vibration for Embedded Carbon Nanotubes," J. Sound Vib., 296, pp. 746-756.

[33] Woinowsky-Krieger, S., 1950, "The Effect of an Axial Force on the Vibration of Hinged Bars," ASME J. Appl. Mech., 17(1), pp. 35-36.

[34] Cheung, Y. K., and Lau, S. L., 1982, "Incremental Time-Space Finite Strip Method for Non-Linear Structural Vibrations,” Earthquake Eng. Struct. Dyn., 10(2), pp. 239-253.

[35] Treacy, M. M. J., Ebbesen, T. W., and Gibson, J. M., 1996, "Exceptionally High Young's Modulus Observed for Individual Carbon Nanotubes," Nature, 381, pp. 678-680.

[36] Wang, Z. L., Gao, R. P., Poncharal, P., de Heer, W. A., Dai, Z. R., and Pan, Z. W., 2001, "Mechanical and Electrostatic Properties of Carbon Nanotubes and Nanowires," Mater. Sci. Eng. C, 16(1-2), pp. 3-10.

[37] Wang, C. M., Tan, V. B. C., and Zhang, Y. Y., 2006, "Timoshenko Beam Model for Vibration Analysis of Multi-Walled Carbon Nanotubes," J. Sound Vib., 294(4-5), pp. 1060-1072.

[38] Li, C. Y., and Chou, T. W., 2003, "A Structural Mechanics Approach for the Analysis of Carbon Nanotubes,” Int. J. Solids Struct., 40(10), pp. 2487-2499.

[39] Xiao, J. R., Gama, B. A., and Gillespie, J. W., 2005, "An Analytical Molecular Structural Mechanics Model for the Mechanical Properties of Carbon Nanotubes," Int. J. Solids Struct., 42(11-12), pp. 3075-3092.

[40] Yoon, J., Ru, C. Q., and Mioduchowski, A., 2003, "Vibration of an Embedded Multiwall Carbon Nanotube," Compos. Sci. Technol., 63(11), pp. 1533-1542.

[41] Wang, Q., 2005, "Wave Propagation in Carbon Nanotubes Via Nonlocal Continuum Mechanics," J. Appl. Phys., 98(12), p. 124301 